\title{
First-time administration of the Sydney Melancholia Prototype Index (SMPI) to non-English-speaking patients: a study from Brazil
}

\author{
Mateus F. Messinger, ${ }^{1,2}$ iD Marco A. Caldieraro, ${ }^{1,2}$ (iD Bruno P. Mosqueiro,,$^{1,2}$ (iD Felipe B. \\ da Costa, ${ }^{1,2}$ iD William dos S. Barcelos, ${ }^{1,2}$ Pedro V. Santos, ${ }^{1,2}$ Gordon Parker, ${ }^{3}$ Marcelo P. Fleck ${ }^{1,2}$ \\ ${ }^{1}$ Programa de Pós-Graduação em Psiquiatria e Ciências do Comportamento, Universidade Federal do Rio Grande do Sul (UFRGS), Porto \\ Alegre, RS, Brazil. ${ }^{2}$ Serviço de Psiquiatria, Hospital de Clínicas de Porto Alegre, UFRGS, Porto Alegre, RS, Brazil. ${ }^{3}$ School of Psychiatry, \\ University of New South Wales, Sydney, NSW, Australia.
}

\begin{abstract}
Objective: The Sydney Melancholia Prototype Index (SMPI) is a scale that uses a non-conventional strategy to assess melancholia status based on prototypic symptoms and illness course variables. This study aimed to evaluate the performance of the first translation of this instrument in a non-Englishspeaking population.

Methods: A sample comprising 106 Brazilian outpatients with depression was evaluated simultaneously with the Brazilian version of the self-rated SMPI (SMPI-SR) and clinician-rated SMPI (SMPI$\mathrm{CR}$ ). The kappa coefficient and $t$ test were used to evaluate concurrent validity vs. DSM-IV, CORE system, Hamilton Depression Rating Scale-6 item (HAM-D6), and HAM-D17 assignments to a melancholic or non-melancholic class. The prevalence of melancholia as well as sensitivity and specificity were calculated across instruments.

Results: The prevalence of melancholia was highest using DSM-IV criteria (56.6\%). The kappa agreement between SMPI-CR and DSM-IV melancholia assignment was moderate (kappa 0.44, $\mathrm{p} \leqslant 0.001$ ). SMPI-CR-assigned melancholic patients had significantly higher CORE, HAM-D17, and HAM-D6 scores. The test-retest consistency values for the SMPI were modest at best, and somewhat superior for the CR version.

Conclusion: The Brazilian SMPI-CR presented satisfactory psychometric properties (which were superior to those of the SMPI-SR), and therefore appears to be a useful option for identifying melancholia and studying its causes and optimal treatments.
\end{abstract}

Keywords: Depressive disorder/diagnosis; depression; melancholia; sensitivity and specificity; severity of illness index

\section{Introduction}

The term "melancholia" (black bile) has evolved in meaning since it first appeared in the Corpus Hippocraticum (460-370 BC), ${ }^{1}$ until it came to be equated with a certain subtype of depression, namely endogenous depression. ${ }^{2}$ Along the same lines, the notion of depression as a single entity varying along a continuum of severity has been reexamined, with some proponents introducing the notion of melancholia as categorically distinct from a heterogeneous residual group of non-melancholic depressive conditions. As proposed, melancholia is characterized by increased prevalence of genetic and biological determinants, including psychomotor disturbance (PMD), and differential response to physical treatments such as broad action antidepressants and electroconvulsive therapy (ECT) ${ }^{3,4}$ Instruments created to diagnose melancholia

Correspondence: Mateus F. Messinger, Universidade Federal do Rio Grande do Sul, Hospital de Clínicas de Porto Alegre, Serviço de Psiquiatria, Rua Ramiro Barcelos, 2350, sala 400N, CEP 90035-903, Porto Alegre, RS, Brazil.

E-mail: mateusfm@gmail.com

Submitted Oct 01 2020, accepted Dec 29 2020, Epub Mar 262021. include the CORE system, 5,6 the Bech-Rafaelsen Melancholia Scale (MES), ${ }^{7,8}$ and the Salpêtrière Retardation Rating Scale (SRRS). ${ }^{9}$ In addition, a six-item version of the Hamilton Rating Scale for Depression $\left(\right.$ HAM-D6) ${ }^{10}$ has been validated as a measure of melancholia. ${ }^{11-13}$

The CORE is a measure of PMD, ${ }^{14}$ held since classical antiquity to be a key marker of melancholia. It has been validated as a measure of melancholia in a number of studies evaluating causal factors and treatment response. ${ }^{14-17}$ However, the CORE measure is less accurate in young patients with melancholia (in whom PMD may be less severe) and in those who do not present at the nadir of their episode as it weights current and not historical signs. ${ }^{18}$ In light of those limitations, Parker et al. ${ }^{19,20}$ developed the Sydney Melancholia Prototype Index (SMPI) as a diagnostic measure of

How to cite this article: Messinger MF, Caldieraro MA, Mosqueiro BP, da Costa FB, Barcelos WS, Santos PV, et al. First-time administration of the Sydney Melancholia Prototype Index (SMPI) to non-English-speaking patients: a study from Brazil. Braz J Psychiatry. 2021;43:599-604. http://dx.doi.org/10.1590/1516-4446-2020-1542 
melancholia capturing prototypic symptoms and illness correlates associated with melancholia. The original SMPI was based on self-report of depressive experiences (SERDEX) and has both self-report (SMPI-SR) and clinician-report (SMPI-CR) versions. SMPI, as a prototypic instrument, follows Q-sort methodology, which is substantially different from that used by traditional psychometric scales. Briefly, Q-methodology focuses on the viewpoints of participants to uncover different patterns (instead of their numerical distribution among the larger population). ${ }^{21}$

The SMPI lists 12 characteristics of melancholic depression (eight symptoms and four non-symptoms) on the left-hand column, and 12 characteristics of nonmelancholic depression (six symptoms and six nonsymptoms) on the right-hand column. ${ }^{19}$ Both patient and clinician versions involve checking the characteristics they recognize as present when depression is at its worst. Patients with a diagnosis of depression are categorized as melancholic if the number of items ticked on the left column exceeds by at least four the number of items ticked on the right-hand column. The same cutoff score of four or more was derived analytically in three studies, with assignment of melancholic vs. non-melancholic depression being quantified with $80-90 \%$ accuracy. ${ }^{18,19,22}$ Recently, a Brazilian Portuguese version of the SMPI was developed, following a 10-step guideline of the International Society for Pharmacoeconomics and Outcomes Research (ISPOR) task force for translation and cultural adaptation. ${ }^{23}$

Here, we present the results obtained with the initial administration of the Brazilian Portuguese SMPI to a non-English-speaking sample, as well as evaluate some psychometric properties of the instrument in a Brazilian sample. To evaluate the performance of both SMPI-CR and SMPI-SR in acutely depressed patients, we chose the same psychometric properties as applied in the original instrument for a Q-sort methodology scale. Firstly, we examined the agreement and accuracy between SMPI melancholic and non-melancholic prototypes compared to other diagnostic instruments (including DSM-IV). Secondly, we evaluated discriminant validity, comparing SMPI assignments vs. CORE and HAM-D (both the 17item and 6-item versions) scores. Thirdly, we evaluated the adequacy of the cutoff score for SMPI assignment for a melancholia diagnosis. Fourthly, we quantified the reliability of the translated/adapted instrument by undertaking a test-retest study.

\section{Methods}

Data were collected from depressed patients attending a tertiary referral clinic for initial assessment. The SMPI retest component was administered 1-3 weeks after the initial test. All non-self-reported measures were administered by trained psychiatrists.

\section{Participants}

A convenience sample was enrolled, comprising consecutive patients attending a tertiary outpatient mood disorder clinic (Programa de Transtornos de Humor, PROTHUM) at Hospital de Clínicas de Porto Alegre, a general university hospital in Brazil. Participants were enrolled from January 2017 to September 2018. Patients of both sexes, aged 18 years or older who met DSM-IV criteria for a current major depressive episode were included, whether they had unipolar or bipolar disorder. Patients who refused to participate and those who did not meet DSM-IV depression criteria at the time of the evaluation or who were unable to understand or complete the research instruments were excluded.

\section{Procedures}

The Mini International Neuropsychiatric Interview-PLUS (MINI-PLUS 5.0) (the most recent version available in Brazilian Portuguese) was administered for a current depression diagnosis, including melancholia and psychiatric co-morbidities according to DSM-IV criteria.

The SMPI-SR and SMPI-CR were administered in a standardized manner before the MINI-PLUS and CORE measures. In addition to rating all 24 SMPI items, patients and clinicians checked a five-point SMPI appendix that allows the rater to record how well (on a 1-5 scale) the patient matches the melancholic or non-melancholic prototype ("Now evaluate to what extent you believe that one of the categories above best represents your overall profile"). Retests were performed by different raters 1 to 3 weeks after the test.

The HAM-D17 was used at baseline to quantify depression severity, and allowed HAM-D6 ${ }^{12}$ scores to be generated. This latter subscale is composed of items $1,2,7,8,10$, and 13 from the HAM-D17 and is considered more adequate than the HAM-D17 to assess nuclear melancholic symptoms of depression. ${ }^{10}$

The CORE measure required the clinician to rate 18 signs of PMD at baseline, with each item scored on a 0-3 scale. A total score greater than or equal to 8 indicates melancholic depression.

\section{Statistical analysis}

Data analyses were processed using SPSS version 19. The diagnostic accuracy of different tests to detect melancholia was compared using the kappa statistic. Quantitative analysis of HAM-D and CORE scores were performed using $t$ tests. The DSM-IV melancholia specifier and the CORE measure were provisionally used as gold standards for diagnosis of melancholia to assess the diagnostic accuracy of the SMPI. The test-retest analysis also used the kappa coefficient.

\section{Ethics statement}

The study protocol was approved by the research ethics committee at Hospital de Clínicas de Porto Alegre (number 16-0540). All patients signed an informed consent prior to entering the study.

\section{Results}

The sample consisted of 106 individuals, of whom $79.2 \%$ were women, as described in Table 1. The overall 
prevalence rates of bipolar disorder and lifetime psychotic features (as diagnosed by the assessing clinician) were $20.9 \%$ and $32.4 \%$, respectively.

The prevalence of melancholia according to different criteria is presented in Table 2. For comparison, Table 2 also shows the prevalence of melancholia observed in an Australian sample reported by Parker et al. ${ }^{18}$ in the original SMPI study. In both analyses, the DSM-IV melancholia specifier gave the highest percentage of melancholic diagnoses, while the SMPI-SR generated the lowest rate.

Table 3 compares the responses provided by patients and clinicians when answering the SMPI appendix question. Clinicians whose SMPI-CR ratings generated a diagnosis of melancholia assigned $100 \%$ of patients to options 1 or 2 (reflecting the view that the episode was most likely to be melancholic in type). Patients whose SMPI-SR rating was compatible with melancholia were most likely to affirm options 1 or 2 (81\%), while the remaining 19\% affirmed option 3 (i.e., the depression was indeterminate in type). Patients with a non-melancholic SMPI-SR rating were more likely $(62.5 \%)$ to affirm option 3 , while $55.6 \%$ of those with a non-melancholic SMPI-CR rating were assigned to options 4 and 5 (weighting nonmelancholic depression); an additional $22.2 \%$ were assigned to the indeterminate option 3.

Table 4 examines the agreement between SMPI-SR or SMPI-CR vs. the complementary SMPI scale and other measures of melancholia (i.e., SMPI-SR vs. SMPI-CR, DSM-IV melancholia specifier, and CORE measure with a cutoff of 8 points or more ${ }^{5}$; and SMPI-CR vs. SMPI-R,

Table 1 Demographic data $(n=106)$

\begin{tabular}{lc}
\hline Characteristic & \\
\hline Female gender, $\mathrm{n}(\%)$ & $84(79.2)$ \\
Age, mean (SD) & $51.1(11.9)$ \\
Years of schooling, mean (SD) & $8.6(3.8)$ \\
Economic class, $\mathrm{n}(\%)^{*}$ & \\
B & $11(10.4)$ \\
C & $76(71.7)$ \\
D, E & $19(17.9)$ \\
& \\
DSM-IV diagnosis, $\mathrm{n}(\%)$ & $22(20.9)$ \\
Bipolar disorder & $34(32.4)$ \\
Lifetime psychosis & \\
\hline SD $=$ standard deviation. & \\
* Economic class classification according to Brazil 2018 Economic \\
Classification Criteria of the Associação Brasileira de Empresas de \\
Pesquisa (ABEP). Class A corresponding to highest income and \\
class E to lowest.
\end{tabular}

DSM-IV melancholia specifier, and CORE measure with a cutoff of 8 points or more ${ }^{5}$ ). To evaluate discriminant validity, we compared the means of the CORE, HAMD17, and HAM-D6 obtained for patients diagnosed with both melancholic and non-melancholic depression according to SMPI.

The SMPI-CR assignment to melancholia had moderate agreement with the CORE assignment to melancholia and with the DSM-IV melancholia criterion. Regarding discriminant validity, the melancholic group defined by SMPI-CR had significantly greater PMD as measured by the CORE than the non-melancholic group (mean scores 8.6 vs. $5.0, p<0.001$ ) with an effect size of 0.83 . When we compared the HAM-D scores of SMPI-CR-assigned melancholic subjects (vs. non-melancholic subjects), there was a larger effect size for the HAM-D6 (0.65) than for the HAM-D17 measure (0.47). This finding indicates greater depressive severity in the SMPI-CR-assigned melancholic subjects. The percentage of patients diagnosed with melancholia by SMPI and also meeting the CORE $\geqslant 8$ criteria was $50 \%$ for SMPI-SR and $55 \%$ for SMPI-CR.

The SMPI-SR assignment of melancholia did not agree statistically with melancholia assignment by either CORE or DSM-IV criteria, although there was a $50 \%$ concordance in melancholia assignment by SMPI-SR and CORE $\geqslant$ 8. SMPI-SR-melancholic-defined subjects returned higher HAM-D6 scores than non-melancholic subjects, but there were no differences in HAM-D17 and CORE total scores between the two groups. The agreement between SMPI-SR and SMPI-CR was low.

Table 5 shows SMPI sensitivity and specificity data (for assigning melancholic status) in the presence of DSM-IV or CORE diagnoses as reference measures. Despite the low sensitivity, there was high specificity for SMPI-CR (0.96) in relation to DSM-IV assignment and moderate specificity in relation to CORE $\geqslant 8(0.79)$. In relation to DSM-IV and CORE $\geqslant 8$, the SMPI-SR also showed low sensitivity (23.3 and $33.3 \%$, respectively), but moderate specificity (84.8 and $80.6 \%$, respectively).

The test-retest results showed significant agreement for both versions of the SMPI. Specifically, the SMPI-CR had low to moderate kappa coefficient agreement of 0.39 ( $p=0.003$ ), while the SMPI-SR had a low kappa coefficient of $0.27(p=0.045)$.

\section{Discussion}

The SMPI has excellent face validity, being composed of features that have been classically associated with

Table 2 Prevalence of melancholia according to different criteria in the current and in the study by Parker et al. ${ }^{18}$

\begin{tabular}{lcc}
\hline & & Prevalence $(\%)$ \\
\cline { 2 - 3 } Melancholia criterion & Current study $(\mathrm{n}=106)$ & Parker et al. ${ }^{18}(\mathrm{n}=214)$ \\
\hline DSM-IV specifier & 56.6 & 64.5 \\
CORE system $\geqslant 8^{*}$ & 31.4 & - \\
SMPI-SR & 19.8 & 37.0 \\
SMPI-CR & 31.1 & 44.5 \\
\hline
\end{tabular}

* CORE system $\geqslant 8$ = cutoff point for melancholia.

SMPI-CR = Sydney Melancholia Prototype Index - clinician-rated version; SMPI-SR = Sydney Melancholia Prototype Index - self-report version. 

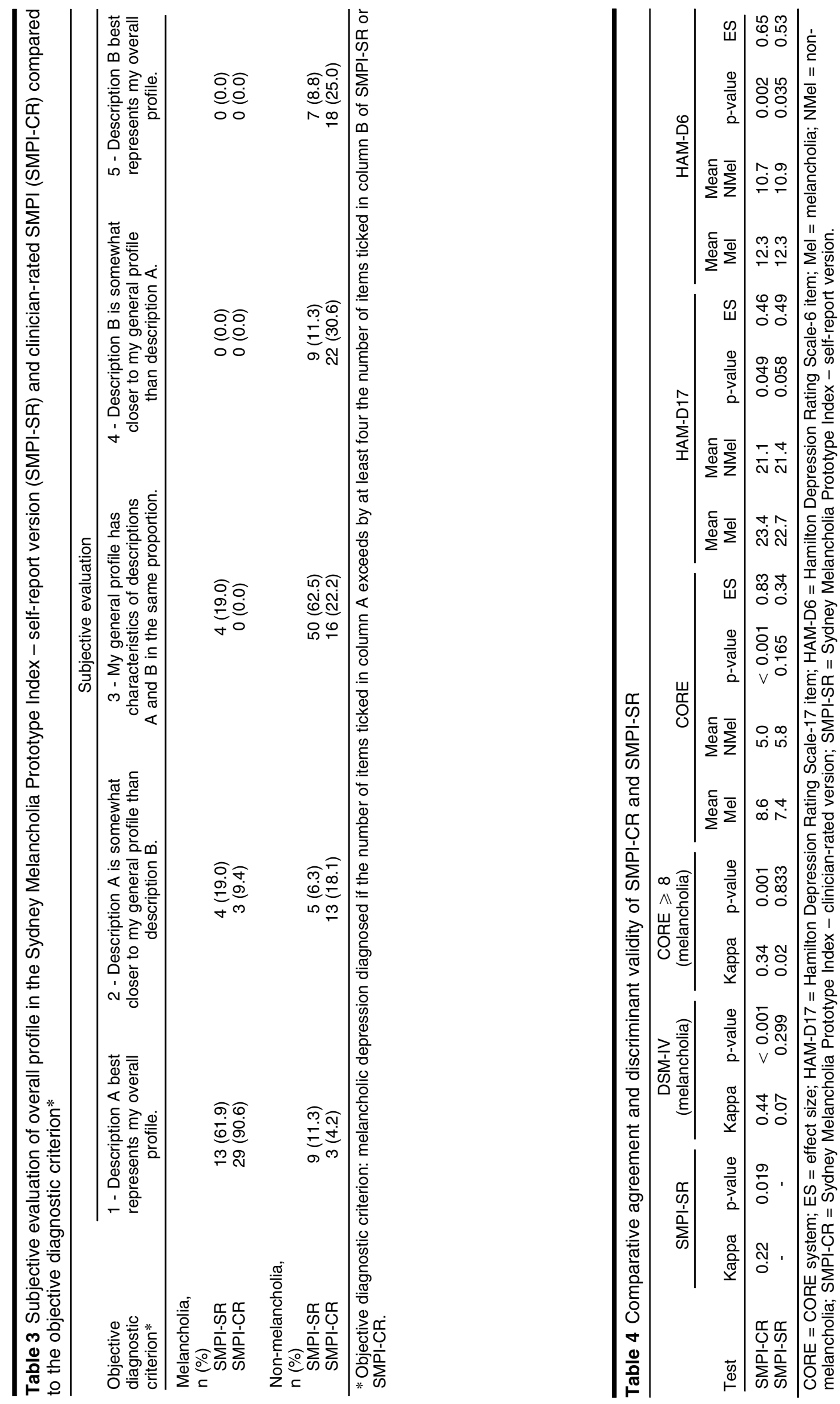
Table 5 Sensitivity and specificity of SMPI measures in patients meting DSM-IV and CORE criteria for melancholia

\begin{tabular}{lcccc}
\hline & \multicolumn{2}{c}{ DSM-IV } & \multicolumn{2}{c}{ CORE $\geqslant 8$} \\
\cline { 2 - 3 } Test & Sensitivity & Specificity & Sensitivity & Specificity \\
\hline SMPI-CR & 51.7 & 95.6 & 54.5 & 79.2 \\
SMPI-SR & 23.3 & 84.8 & 33.3 & 80.6 \\
\hline
\end{tabular}

Data presented as percentage.

SMPI-CR = Sydney Melancholia Prototype Index - clinician-rated version; SMPI-SR = Sydney Melancholia Prototype Index - self-report version.

melancholia, such as low energy, anhedonia, non-reactive mood, morning worsening, lack of future perspective, motor slowing, lack of concentration, weight loss, severity disproportionate to external events, absence of significant stressors in childhood, good interpersonal patterns between episodes, and an absence of triggering factors. When administering the SMPI, it is important to keep in mind that the most severe episode throughout the patient's life (not necessarily the current episode) should be considered. Another relevant point is the prototypic system of differentiating melancholic from non-melancholic depression - the SMPI is not a conventional scale generating a score that reflects the intensity of the construct.

Considering the different criteria used to assign melancholia in the study, the DSM-IV specifier for melancholia identified the highest prevalence (56.6\%). This finding is consistent with the fact that the DSM-IV specifier criteria are very broad and almost overlap the criteria for major depression, ${ }^{24}$ thus risking over-diagnosing melancholia. The SMPI-SR generated the lowest prevalence of melancholia (19.8\%), followed by the SMPI$\mathrm{CR}$ and the CORE measure with very similar values ( $31 \%$ for both). Similar to the CORE measure, the SMPI showed more restrictive criteria for melancholia. Such data suggest that the SMPI identifies a more circumscribed and homogeneous group of patients, which supports the development of studies to find biological correlates and more specific treatments.

There were some similarities between our study and the Australian study undertaken by Parker et al. ${ }^{18}$ In both, DSM criteria generated the highest prevalence of melancholia and the SMPI-SR generated the lowest rate. However, there was a lower prevalence of melancholia using different instruments in the Brazilian sample, which may reflect different referral patterns, with a higher proportion of melancholic patients referred to the Australian facility.

The SMPI-CR generated moderate kappa coefficients according to other melancholia criteria (DSM-IV and CORE $\geqslant 8$ specifier). It also presented larger effect sizes when melancholic vs. non-melancholic subjects were compared based on scales that measure intensity of melancholic symptoms (CORE and HAM-D6), supporting its convergent validity. The self-report version (SMPI-SR) did not correlate with the other measures (DSM specifier, HAM-D17, and CORE $\geqslant 8$ ), but it was associated with higher HAM-D6 for melancholic patients. In addition, the diagnostic agreement of melancholia evaluated by SMPI$\mathrm{SR}$ and SMPI-CR was low, which is consistent with the results described by Parker et al. $^{18}$ in an Australian sample. Such findings argue for favoring the CR above the SR version of the SMPI.

The SMPI was developed to assess the patient's experience of depression on a lifetime basis. Therefore, it should yield similar results if administrated at different time points. In this study, we quantified higher test-rest reliability for the CR than for the SR version, but with kappa coefficients being modest at best.

One limitation of this study is the current lack of a goldstandard criterion for the diagnosis of melancholia, which has been a recent subject of discussion. ${ }^{25}$ In the study by Parker et al., ${ }^{18}$ a clinical interview by experts was used as the diagnostic reference standard. Since the clinical interview was the gold standard for the development of the scale, we used a different strategy comparing the performance of SMPI with that of other classical instruments used in literature to evaluate melancholia. Consequently, we assessed convergent validity rather than criterion validity. A second limitation is that the sample comprised patients with chronic resistant depression, attested by the fact that they reached a tertiary outpatient center. Another limitation considering prototypic scales is that all psychometric properties are based on individual item scores or total scores, particularly scalability; it was not possible to perform factor analysis or evaluations of internal consistency and unidimensionality.

In conclusion, this study presents the first results of administration of the SMPI instrument in a language other than English. The Brazilian version of the SMPI has excellent face validity. The SMPI-CR version performed satisfactorily in discriminating melancholia when compared to other instruments. These results indicate that the Brazilian SMPI-CR is suitable for use on future studies on melancholia, with the potential to characterize a more homogeneous group of individuals with a melancholic syndrome. The SMPI-SR performance was poorer, and future studies are needed to clarify if this self-report version can be adequately used in Brazilian samples.

\section{Acknowledgements}

This study received specific funding from Fundo de Incentivo à Pesquisa e Eventos/Hospital de Clínicas de Porto Alegre (FIPE/HCPA).

\section{Disclosure}

The authors report no conflicts of interest. 


\section{References}

1 Ban TA. From melancholia to depression: a history of diagnosis and treatment [Internet]. 2014 [cited 2021 Jan 21]. inhn.org/fileadmin/ previews_new/From_Melancholia_to_Depression_March_6_2014.pdf

2 Telles-Correia D, Marques JG. Melancholia before the twentieth century: fear and sorrow or partial insanity? Front Psychol. 2015;6:81.

3 Parker G. Defining melancholia: the primacy of psychomotor disturbance. Acta Psychiatr Scand Suppl. 2007;115:21-30.

4 Fink M, Bolwig TG, Parker G, Shorter E. Melancholia: restoration in psychiatric classification recommended. Acta Psychiatr Scand. 2007;115:89-92.

5 Hadzi-Pavlovic D, Hickie I, Brodaty H, Boyce P, Mitchell P, Wilhelm $\mathrm{K}$, et al. Inter-rater reliability of a refined index of melancholia: the CORE system. J Affect Disord. 1993;27:155-62.

6 Hickie I, Mason C, Parker G. Comparative validity of two measures of psychomotor function in patients with severe depression. $J$ Affect Disord. 1996;37:143-9.

7 Bech P. The Bech-Rafaelsen Melancholia Scale (MES) in clinical trials of therapies in depressive disorders: a 20-year review of its use as outcome measure. Acta Psychiatr Scand. 2002;106:252-64.

8 Bech P, Rafaelsen OJ. The use of rating scales exemplified by a comparison of the Hamilton and the Bech-Rafaelsen Melancholia Scale. Acta Psychiatr Scand. 1980;62:128-32.

9 Dantchev N, Widlöcher DJ. The measurement of retardation in depression. J Clin Psychiatry. 1998;59 Suppl 14:19-25.

10 Bech P, Allerup P, Gram LF, Reisby N, Rosenberg R, Jacobsen O, et al. The Hamilton Depression Scale. Evaluation of objectivity using logistic models. Acta Psychiatr Scand. 1981;63:290-9.

11 Hooper CL, Bakish D. An examination of the sensitivity of the six-item Hamilton Rating Scale for depression in a sample of patients suffering from major depressive disorder. J Psychiatry Neurosci. 2000;25:178-84.

12 Caldieraro MA, Vares EA, Spanemberg L, Becker FR, Fleck MP. Association between core-assigned melancholia and the melancholia subscale of the HAM-D. J Affect Disord. 2014;172:175-8.

13 Thase ME, Hersen M, Bellack AS, Himmelhoch JM, Kupfer DJ. Validation of a Hamilton subscale for endogenomorphic depression. J Affect Disord. 1983;5:267-78.
14 Parker G, McCraw S. The properties and utility of the CORE measure of melancholia. J Affect Disord. 2017;207:128-35.

15 Caldieraro MA, Baeza FL, Pinheiro DO, Ribeiro MR, Parker G, Fleck MP. Clinical differences between melancholic and nonmelancholic depression as defined by the CORE system. Compr Psychiatry. 2013;54:11-5.

16 Caldieraro MA, Baeza FL, Pinheiro DO, Ribeiro MR, Parker G, Fleck MP. Prevalence of psychotic symptoms in those with melancholic and nonmelancholic depression. J Nerv Ment Dis. 2013;201: 855-9.

17 Spanemberg L, Caldieraro MA, Vares EA, Wollenhaupt-Aguiar B Kauer-Sant'Anna M, Kawamoto SY, et al. Biological differences between melancholic and nonmelancholic depression subtyped by the CORE measure. Neuropsychiatr Dis Treat. 2014;10:1523-31.

18 Parker G, Mccraw S, Fletcher K, Friend P, Futeran S. Validation of a new prototypic measure of melancholia. Compr Psychiatry. 2013;54:835-41.

19 Parker G, Mccraw S, Blanch B, Hadzi-Pavlovic D, Synnott H, Rees AM. Discriminating melancholic and non-melancholic depression by prototypic clinical features. J Affect Disord. 2013;144:199-207.

20 Parker G, Fletcher K, Hyett M, Hadzi-Pavlovic D, Barrett M, Synnott $\mathrm{H}$. Measuring melancholia: the utility of a prototypic symptom approach. Psychol Med. 2009;39:989-98.

21 Valenta AL, Wigger U. Q-methodology: definition and application in health care informatics. J Am Med Inform Assoc. 1997;4:501-10.

22 Parker G, McCraw S, Hadzi-Pavlovic D. The utility of a classificatory decision tree approach to assist clinical differentiation of melancholic and non-melancholic depression. J Affect Disord. 2015;180: 148-53.

23 Messinger MF, Caldieraro MA, Mosqueiro BP, da Costa FB, Possebon GM, Nascimento Santos PV, et al. Sydney Melancholia Protoype Index (SMPI): translation and cross-cultural adaptation into Brazilian Portuguese. Trends Psychiatry Psychother. 2020;42: 247-55.

24 Parker G, Brotchie $\mathrm{H}$. [Major depression invites major concerns]. Braz J Psychiatry. 2009;31 Suppl 1:S3-6.

25 Carrozzino D, Patierno C, Fava GA, Guidi J. The Hamilton Rating Scales for depression: a critical review of clinimetric properties of different versions. Psychother Psychosom. 2020;89:133-50. 